\title{
The development and performance evaluation of a hybrid photo-detector for Hyper-Kamiokande
}

\author{
Miao JIANG* \\ Department of Physics, Kyoto University, Sakyo-ku, Kyoto 606-8502, Japan \\ E-mail: mjiangescphys.kyoto-u.ac.jp
}

Yasuhiro NISHIMURA ${ }^{a}$, Yuji OKAJIMA ${ }^{b}$, Masahiro $\mathrm{KUZE}^{b}$, Yusuke SUDA ${ }^{c}$, Ryosuke AKUTSU $^{c}$, Daisuke FUKUDA ${ }^{d}$, Seiko HIROTA, Masashi YOKOYAMA ${ }^{c}$, Masayuki NAKAHATA $^{a}$, Masato SHIOZAWA ${ }^{a}$, Yoshinari HAYATO ${ }^{a}$, Shoei NAKAYAMA ${ }^{a}$, Hidekazu TANAKA ${ }^{a}$, Tsuyoshi NAKAYA, Akihiro MINAMINO, Akimichi TAKETA ${ }^{e}$, Yoshihiko KAWAI $^{f}$, Takayuki OHMURA ${ }^{f}$, Masatoshi SUZUKI ${ }^{f}$

${ }^{a}$ Institute for Cosmic Ray Research, University of Tokyo, Kashiwa, Chiba 277-8582, Japan

${ }^{b}$ Department of Physics, Tokyo Institute of Technology, Meguro-ku, Tokyo 152-8551, Japan

${ }^{c}$ Department of Physics, University of Tokyo, Bunkyo-ku, Tokyo 113-0033, Japan

${ }^{d}$ Department of Physics, Okayama University, Okayama, Okayama 700-8530, Japan

${ }^{e}$ Earthquake Research Institute, University of Tokyo, Bunkyo-ku, Tokyo 113-0032, Japan

${ }^{f}$ Hamamatsu Photonics K.K., Hamamatsu, Shizuoka, 430-8587, Japan

\begin{abstract}
We have been developing a new hybrid photo-detector (HPD) for Hyper-Kamiokande, which is a proposed next generation megaton-class water Cherenkov detector. The prototypes of the 50 $\mathrm{cm}$ HPD with $\phi 5 \mathrm{~mm}$ avalanche diode (AD) [1] and the $50 \mathrm{~cm}$ HPD with $\phi 20 \mathrm{~mm}$ AD (R12850, Hamamatsu Photonics K.K.) were designed and produced after successful fabrication of $20 \mathrm{~cm}$ HPD. For the $50 \mathrm{~cm}$ HPD with $\phi 5 \mathrm{~mm} \mathrm{AD}$, the transit time spread, linearity and temperature dependence of gain have been evaluated. For our target design of the $50 \mathrm{~cm}$ HPD with $\phi 20 \mathrm{~mm}$ $\mathrm{AD}$, two solutions by the segmented $\mathrm{AD}$ and the transformer coupling were tried to suppress the capacity seen by preamplifier since it is difficult to design a high speed preamplifier with low noise level for a large area AD due to the large junction capacitance. They successfully reduced the noise, and the performance of HPD showed significant improvements.
\end{abstract}

International Conference on New Photo-detectors - PhotoDet 2015

6-9 July 2015

Moscow, Troitsk, Russia

${ }^{*}$ Speaker. 


\section{Hyper-Kamiokadande Project}

Hyper-Kamiokande (Hyper-K) is a next generation megaton-class water Cherenkov detector (Fig. 1) [2]. It has rich physics targets such as search for proton decay, study of neutrino properties using neutrino oscillation, detection and study of astrophysical neutrinos. Its total (fiducial) water mass of $0.99(0.56)$ million tons would be approximately 20 (25) times as large as that of SuperKamiokande (Super-K) [3]. In the baseline design, for the inner detector and the outer detector, $99,000 \phi 50 \mathrm{~cm}$ photosensors and 25,000 $\phi 20 \mathrm{~cm}$ photosensors are needed, respectively. The photocoverage of the inner detector is $20 \%$ which is a half of that of Super-K.

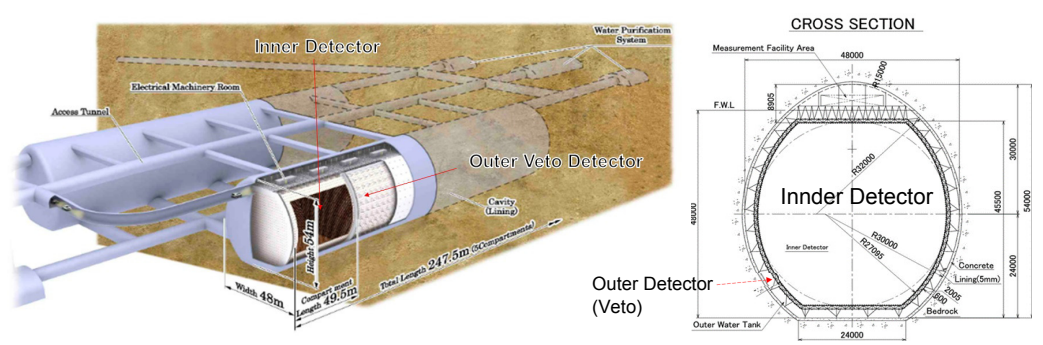

Figure 1: The schematic view (left) and cross section view (right) of the Hyper-Kamiokande detector.

\section{Development of Hybrid Photo-Detector for Hyper-K}

A large aperture hybrid photo-detector (HPD), which is a hybrid of a vacuum tube and an avalanche diode (AD), is under development for Hyper-K. The principle of HPD is shown in Fig. 2. The photo-electron produced at the photocathode is accelerated by a high electric field between the photocathode and the $\mathrm{AD}$, then multiplied by the avalanche multiplication process at a high bias voltage in the AD. A preamplifier is employed to gain the observed signal further (Fig. 3). Because of the shorter drift path in the AD than that in the dynodes of a photomultiplier tube (PMT) and the high gain at the first amplification stage, the HPD has better timing and charge resolution than those of the PMT.

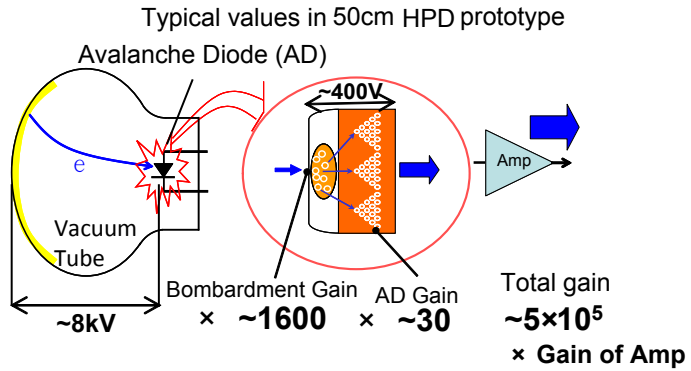

Figure 2: Principle of the HPD amplification and typical values of the $50 \mathrm{~cm}$ HPD.

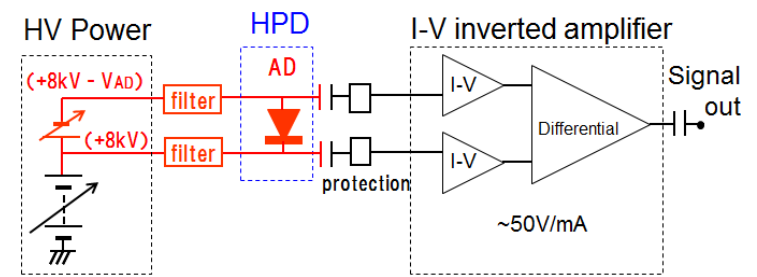

Figure 3: The schematic view of the circuit in the HPD.

We developed three kinds of HPDs in the order of difficulty as follows.

1. $20 \mathrm{~cm}$ HPD with $\phi 5 \mathrm{~mm}$ AD

The prototypes are being tested in a 200 tons water tank after performance evaluation [1]. 
2. $50 \mathrm{~cm}$ HPD with $\phi 5 \mathrm{~mm} \mathrm{AD}$

The prototype of this kind of HPD was developed and evaluated firstly to test the large diameter HPD production technology. The $\phi 5 \mathrm{~mm}$ AD allows the sharing of the preamplifier design with the $20 \mathrm{~cm}$ HPD's.

\section{3. $50 \mathrm{~cm}$ HPD with $\phi 20 \mathrm{~mm}$ AD}

The $50 \mathrm{~cm}$ HPD with a $\phi 20 \mathrm{~mm}$ AD and its circuit were developed and evaluated. On the issue of the large $\mathrm{AD}$ junction capacitance, two proposed solutions, the segmented $\mathrm{AD}$ and the transformer coupling have been tested.

\section{Performance evaluation of $50 \mathrm{~cm}$ HPD with $\phi 5 \mathrm{~mm}$ AD}

\subsection{Signal response of single photoelectron}

The signal response of single photoelectron (p.e.) of $50 \mathrm{~cm}$ HPD with $\phi 5 \mathrm{~mm}$ AD has been evaluated and summarized in Table 1 [1]. The single p.e. resolution and ratio of peak to valley of HPD is better than that of Super-K PMT (R3600, Hamamatsu Photonics K.K.). The HPD itself has faster response: the $20 \mathrm{~cm}$ HPD with $\phi 5 \mathrm{~mm}$ AD had a rise time of $1.7 \mathrm{~ns}$ and a fall time of $2.7 \mathrm{~ns}$ when no preamplifier was employed.

\subsection{Transit time spread}

A good time resolution is important for the event reconstruction in Hyper-K. The time resolution of a photo-sensor is evaluated by the transit time spread (TTS), which is temporal fluctuation from photon emission to signal appearance. The signal time is measured when the output signal reaches a certain threshold level ( 0.5 p.e. for HPD, 0.25 p.e. for PMT), referring to the laser diode emission time measured according to the synchronization output with a precision less than $0.01 \mathrm{~ns}$.

\begin{tabular}{|c|c|c|}
\hline & $\begin{array}{l}50 \mathrm{~cm} \mathrm{HPD} \\
\mathrm{w} / \phi 5 \mathrm{~mm} \mathrm{AD}\end{array}$ & $\begin{array}{l}\text { Super-K } \\
\text { PMT [1] }\end{array}$ \\
\hline Rise time $(\mathrm{ns})^{1}$ & 7.4 & 10.6 \\
\hline Fall time $(\mathrm{ns})^{2}$ & 11.5 & 13.1 \\
\hline $\begin{array}{l}\text { Pulse width } \\
(\text { FWHM) (ns) }\end{array}$ & 17.1 & 18.5 \\
\hline 1 p.e. resolution $(1 \sigma)^{3}$ & $16 \%$ & $53 \%$ \\
\hline Peak / valley ratio ${ }^{4}$ & 3.9 & 2.2 \\
\hline
\end{tabular}

Table 1: Signal response comparison between HPD and PMT. [1]

1: The $10 \%$ to $90 \%$ rise time. ${ }^{2}$ : The $90 \%$ to $10 \%$ fall time.

3: The ratio of $1 \sigma$ width to the peak value of the 1 p.e. signal in the charge distribution.

4. The height ratio of 1 p.e. peak to the valley between 1 p.e. peak and pedestal peak in the charge distribution.

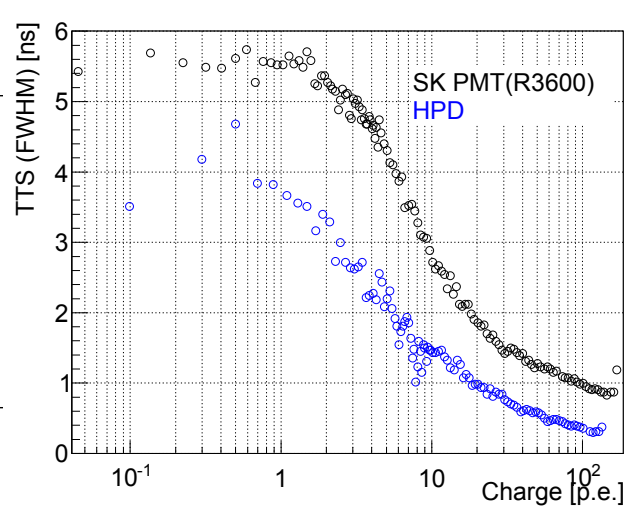

Figure 4: The TTS of the HPD and the SuperK PMT as functions of pulse intensities with the time walk corrected by charge. The light is incident into the center of HPD cathode with a beam diameter less than $1 \mathrm{~mm}$.

Fig. 4 shows the TTS of the HPD as a function of pulse intensities with the time walk corrected by charge. Obviously, the TTS of the HPD is better than that of the Super-K PMT in the full range. 
Ideally the intrinsic resolution of the HPD at single p.e. shows $0.75 \mathrm{~ns}$ at FWHM as indicated by the simulation. Currently, the measured resolution is still limited by the preamplifier design with the slow response and a certain fluctuation on the baseline.

\subsection{Linearity and dynamic range}

The linearity of the HPD's output charge was measured by comparing with the light intensity. In a high light intensity, the AD or the preamplifier might be saturated and the output of HPD would be deviated from the linearity. The photosensor in Hyper-K should have a good sensitivity in the range from 1 to 1,000 p.e. because the Hyper-K detector needs to observe the events in a wide dynamic range: from several $\mathrm{MeV}$ to several $\mathrm{GeV}$.

The linearity of HPD in high light intensity is confirmed by the comparison of the output of two coincident lights and the sum of the separated light using two light sources. By changing the light intensity of the light source, the linearity could be evaluated in a wide range. Comparing with the method used in [4], multiple light sources can measure the linearity without bias because there is no other photo-sensor for reference.

Fig. 5 shows the output charge as a function of the number of the input photoelectrons. It is confirmed that the linearity holds up to around 140 p.e., with the maximum drift of $6 \%$, which is limited by the maximum output voltage (about $800 \mathrm{mV}$ ) of the preamplifier. It will be improved in the future by the redesign of the preamplifier.

\subsection{Temperature dependency of gain}

The temperature dependency of gain is evaluated in a thermostatic room (Fig. 6). The gain temperature coefficient is about $-2.1 \% /{ }^{\circ} \mathrm{C}$ at $20^{\circ} \mathrm{C}$. Since the water temperature of Hyper-K is expected to be stable at $13{ }^{\circ} \mathrm{C}$ in the order of $0.1{ }^{\circ} \mathrm{C}$, the stability of HPD in operation will not be influenced by the thermal factor. Furthermore, this result can also be used to estimate HPD's performance in Hyper-K tank according to the performance in the laboratory where the temperature is $24{ }^{\circ} \mathrm{C}$.
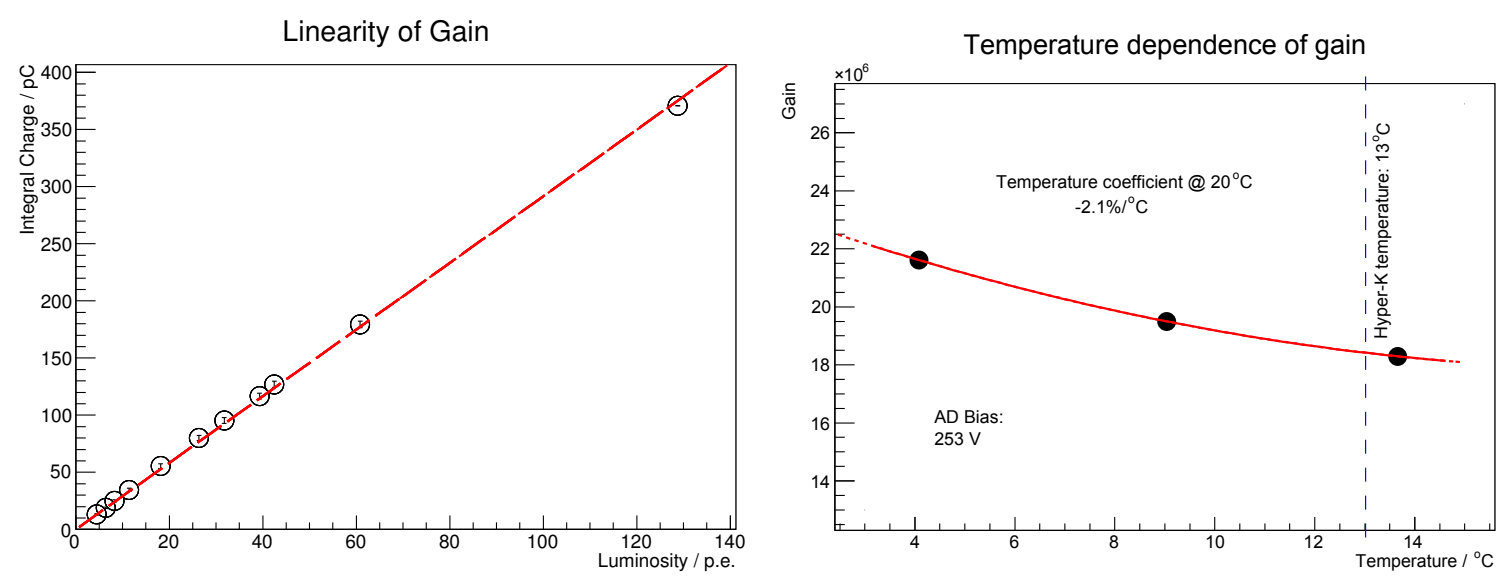

Figure 5: Relationship between output charge and the light intensity. Measured at $8 \mathrm{kV}$ with $293 \mathrm{~V}$ of AD bias.

Figure 6: Temperature dependency of gain of $50 \mathrm{~cm}$ HPD with $\phi 5 \mathrm{~mm}$ AD. Measured at $8 \mathrm{kV}$ with $253 \mathrm{~V}$ of AD bias. 


\section{Research and development of $50 \mathrm{~cm}$ HPD with large area AD}

As has been stated above, a larger area AD is desired for $50 \mathrm{~cm}$ HPD to ensure a high collection efficiency (CE), which is defined as the efficiency to collect the photoelectrons on the AD surface from photocathode. According to the result provided by Hamamatsu, a single channel $\phi 20 \mathrm{~mm}$ AD can make the $\mathrm{CE}$ reach to about $93.3 \%$ while the $\mathrm{CE}$ of a $\phi 5 \mathrm{~mm} \mathrm{AD}$ is less than $10 \%$. However, with the increasing area of $\mathrm{AD}$, the junction capacitance also becomes larger: about $800 \mathrm{pF}$ for $\phi 20$ $\mathrm{mm} \mathrm{AD}$, while about $60 \mathrm{pF}$ for $\phi 5 \mathrm{~mm} \mathrm{AD}$. A larger junction capacitance leads to a higher noise level, a longer time constant of the readout circuit, therefore to worse energy and time resolution.

Two countermeasures, the application of segmented AD and the transformer coupling, were tried to reduce the noise level.

\subsection{Application of segmented AD}

A prototype of segmented 5-ch $\phi 20 \mathrm{~mm} \mathrm{AD} \mathrm{(Fig.} \mathrm{7)} \mathrm{was} \mathrm{developed.} \mathrm{Each} \mathrm{channel} \mathrm{is} \mathrm{amplified}$ by an isolated trans-impedance preamplifier and all the signal are summed to the output (Fig. 8). The lower input capacitance makes it easier to develop a high speed preamplifier with low noise level.

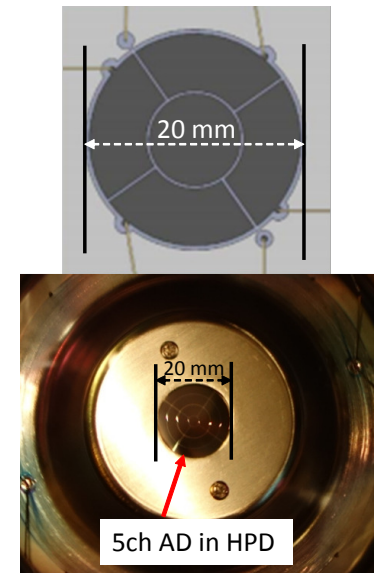

Figure 7: 5-ch $\phi 20 \mathrm{~mm}$ AD.

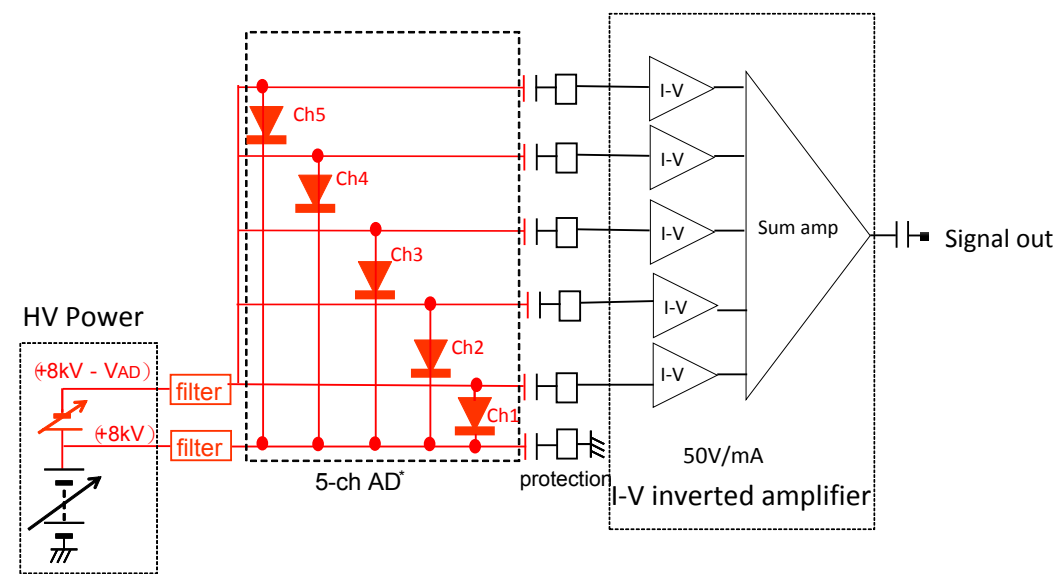

Figure 8: The HPD system with multi-ch AD.

Comparing with the $50 \mathrm{~cm}$ HPD with $\phi 5 \mathrm{~mm}$ AD, the $50 \mathrm{~cm}$ HPD with 5 -ch $\phi 20 \mathrm{~mm}$ AD has a slightly slower response and a worse 1 p.e. resolution (Table 2), which is caused by the large AD junction capacitance. In fact, even though the capacitance in each channel is reduced to 1/5 (i.e. $160 \mathrm{pF})$, it is still larger than that of $\phi 5 \mathrm{~mm} \mathrm{AD}(60 \mathrm{pF})$. Nevertheless, the AD with more channels are not developed because of the difficulty in the pin arrangement and the smaller effective area due to more isolate trench between each channel. Currently, the 1 p.e. resolution of the $50 \mathrm{~cm}$ HPD with 5-ch $\phi 20 \mathrm{~mm}$ AD is better than that of Super-K PMT, although its response is still slower than that of Super-K PMT (Table 2).

\subsection{Application of coupling transformer}

Coupling transformer can be used to suppress the noise gain of the preamplifier by reducing the capacitance seen by the amplifier [5]. The performance of coupling transformer is tested on a 


\begin{tabular}{llll}
\hline & $50 \mathrm{~cm} \mathrm{HPD} \mathrm{w/}$ & $50 \mathrm{~cm} \mathrm{HPD} \mathrm{w/}$ & Super-K PMT[1] \\
& $5-\mathrm{ch} \phi 20 \mathrm{~mm} \mathrm{AD}$ & $\phi 5 \mathrm{~mm} \mathrm{AD}[1]$ & \\
\hline Rise time $(\mathrm{ns})$ & $20.3^{*}$ & 7.4 & 10.6 \\
Fall time $(\mathrm{ns})$ & $21.1^{*}$ & 11.5 & 13.1 \\
Pulse width $(\mathrm{FWHM})(\mathrm{ns})$ & $32.3^{*}$ & 17.1 & 18.5 \\
1 p.e. resolution $(\sigma / \mu)$ & $28 \%$ & $16 \%$ & $53 \%$ \\
Peak / valley ratio & 3.1 & 3.9 & 2.2 \\
\hline
\end{tabular}

Table 2: Comparison of the single photoelectron response of $50 \mathrm{~cm}$ HPD with 5-ch $\phi 20 \mathrm{~mm}$ AD and other detectors. The HPD with 5-ch $\phi 20 \mathrm{~mm}$ AD is measured with a $20 \mathrm{MHz}$ bandwidth filter at $415 \mathrm{~V}$ of AD bias and $8 \mathrm{kV}$ of HV. The result of the HPD with 1-ch $\phi 20 \mathrm{~mm}$ AD is not shown because the single photoelectron pulse can not be seen at $8 \mathrm{kV}$ of $\mathrm{HV}$.

*: The value of the pulse shape after low pass filter.

prototype of the $50 \mathrm{~cm}$ HPD with 1-ch $\phi 14.7 \mathrm{~mm} \mathrm{AD}$ at first.

We compared the pulse shape (Fig. 10), the integral charge distribution (Table 3) of the $50 \mathrm{~cm}$ HPD signal with and without the coupling transformer with a bombardment voltage (HV) of 11 $\mathrm{kV}$. Obviously, the coupling transformer brings a faster response and a better 1 p.e. resolution to the $50 \mathrm{~cm}$ HPD.

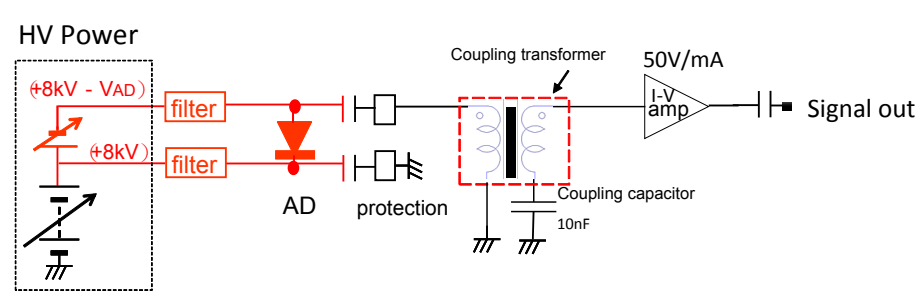

Figure 9: Circuit of the $50 \mathrm{~cm}$ HPD with $\phi 20 \mathrm{~mm} \mathrm{AD}\left(C_{A D}=800\right.$ $\mathrm{pF}$ ) using coupling transformer.

\begin{tabular}{lll}
\hline & w/ trans & w/o trans \\
\hline Rise time (ns) & 13.9 & 19.3 \\
Fall time (ns) & 16.7 & 23.9 \\
Pulse width & 28.8 & 42.2 \\
$($ FWHM) $(\mathrm{ns})$ & & \\
1 p.e. resolution $(\sigma / \mu)$ & $26.9 \%$ & $49.1 \%$ \\
Peak / valley ratio & 4.9 & 1.4 \\
\hline
\end{tabular}

Table 3: Effect of coupling transformer on the multi photoelectron response of $50 \mathrm{~cm}$ HPD w/ $\phi 14.7 \mathrm{~mm}$ AD. Measured at $11 \mathrm{kV}$ with $415 \mathrm{~V}$ of $\mathrm{AD}$ bias and $20 \mathrm{MHz}$ of bandwidth.

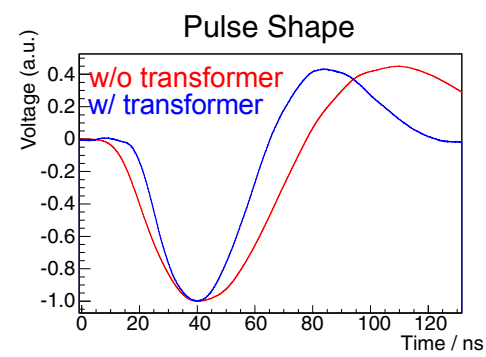

Figure 10: Signal of $50 \mathrm{~cm} \mathrm{HPD}$ with and without transformer.

Considering the safety, a lower HV (e.g. $8 \mathrm{kV}$ ) is preferred although the signal response at a higher HV (e.g. $11 \mathrm{kV}$ ) is better as shown in Fig. 11. However, for the signal response at a HV of 8 $\mathrm{kV}$, a ratio of peak to valley of 1.1 does not satisfy the requirement of Hyper-K, although the single 
p.e. charge resolution is about $51.7 \%$, which is the same level as Super-K PMT. The combined use of the multi-channel $\mathrm{AD}$ and the transformer coupling is expected to give a better performance and will be tested in the future.

The transit time spread of the HPD with coupling transformer at 1 p.e. level is also evaluated to be $6.0 \mathrm{~ns}$ (FWHM) with a HV of $8 \mathrm{kV}$, which is larger than that of Super-K PMT (5.5 ns). As in Section 3.2, this value is also constrained by the preamplifier currently.

The dark count rate, which is defined as the average rate of hit without any incident light, is measured by counting the pulse above threshold level in the waveform recorded by the oscilloscope with a long gate ( $50 \mu s \times 1000$ waveforms). The $50 \mathrm{~cm}$ HPD with $\phi 20 \mathrm{~mm}$ AD (CE: 93.3\%) is estimated to be $9.4 \mathrm{kHz}$ based on the result of the $50 \mathrm{~cm}$ HPD with $\phi 14.7 \mathrm{~mm} \mathrm{AD} \mathrm{(CE:} \mathrm{83 \% ):} 8.33$ $\mathrm{kHz}$. After considering the temperature dependency of dark count rate, the dark rate of $50 \mathrm{~cm}$ HPD with $\phi 20 \mathrm{~mm}$ AD operating under the Hyper-K environment $\left(13^{\circ} \mathrm{C}\right)$ can be reduced to $1 / 2$ (i.e. 4.7 $\mathrm{kHz}$ [6], which is the same level as the Super-K PMT.

\section{Summary}

We have been developing a new hybrid photo-detector for Hyper-Kamiokande. The detailed performance, including linearity, time resolution and temperature dependency of the $50 \mathrm{~cm}$ HPD with $\phi 5 \mathrm{~mm} \mathrm{AD}$, was evaluated. The linearity is confirmed in $6 \%$ up to 140 p.e. Although it does not satisfy the requirement of the photosensor for Hyper-K currently, the performance will be improved with a revised preamplifier since the the linearity range is constrained by the preamplifier output. Furthermore, the time resolution of $50 \mathrm{~cm}$ HPD with $\phi 5 \mathrm{~mm} \mathrm{AD}, 3.6 \mathrm{~ns}$ at 1 p.e. (FWHM), is better than that of Super-K PMT (5.5 ns at 1 p.e.), while it will be also tested again with the new preamplifier after developed. The temperature dependence of gain, $-2.1 \% /{ }^{\circ} \mathrm{C}$ at $20{ }^{\circ} \mathrm{C}$ will have little influence on the stability of the HPD during operation in Hyper-K environment.

Comparing with the $50 \mathrm{~cm}$ HPD with $\phi 5 \mathrm{~mm} \mathrm{AD}$, the $50 \mathrm{~cm}$ HPD with $\phi 20 \mathrm{~mm} \mathrm{AD}$, which is the target design, has a slower signal response and a higher noise level due to the larger capacitance from the larger area of $\mathrm{AD}$. Two solutions, transformer coupling and segmented $\mathrm{AD}$, were found to suppress the noise level and improve the single p.e. resolution effectively. The 50 $\mathrm{cm}$ HPD with $\phi 20 \mathrm{~mm}$ AD showed great potential as a Hyper-Kamiokande photo-detector and the version which can operate in water tank will be ready by the end of 2015 .

\section{References}

[1] Y. Suda, Performance evaluation of new photodetectors for Hyper-Kamiokande, Proceedings of Science, TIPP2014:153, (2014).

[2] K. Abe et al, (The Hyper-Kamiokande working group): Letter of Intent: The Hyper-Kamiokande Experiment Detector Design and Physics Potential, arXiv:1109.3262v1.

[3] Y. Fukuda et al, The Super-Kamiokande Detector, Nuclear Instruments and Methods in Physics Research Section A, 501:418-462, (2003).

[4] Y. Suda, Performance evaluation of the large-aperture Hybrid Photo-Detector, Nuclear Instruments and Methods in Physics Research A, 766:185-188, (2014).

[5] E. Gatti and P.F. Manfredi and D.Marioli, Limitations in transformer coupling between radiation detector and head amplifier, Nuclear Instruments and Methods, 193:539-547, (1982).

[6] M. Jiang et al, Temperature Dependence Measurement of a Hybrid Photo-Detector for Hyper-Kamiokande, JPS Conference Proceedings , 023006 (2015). 